\title{
Rotation Curves of 38 Galaxies in Clusters from Scanning Fabry-Perot observations
}

\author{
P. Amram ${ }^{(1)}$ M. Marcelin(1), C. Balkowski( ${ }^{(2)}$ J. Boulesteix ${ }^{(1)}$, \\ E. le Coarer ${ }^{(3)}$, V. Cayatte ${ }^{(2)}$, W. Sullivan $\operatorname{III}^{(4)}$ \\ (1) Observatoire de Marseille, France \\ (2) Observatoire de Paris-Meudon, France \\ (3) Observatoire de Grenoble, France \\ (4) University of Washington, Seattle, USA
}

\begin{abstract}
A major astrophysical problem is related to the fact that rotation curves (RCs)) of galaxies are flat. The presence of a dark halo is most often invoked to explain that. Some controversies exist concerning the existence of a dark halo for galaxies in very hostile environments like in center of clusters. Using a scanning Fabry-Perot interferometer, we have observed $\mathrm{H} \alpha$ velocity fields of a sample of 38 galaxies located in 7 different clusters of galaxies. From this sample, our conclusion is that spirals located in the central part of clusters do not have decreasing RCs within the optical radius.
\end{abstract}

\section{Introduction}

Many effects of the cluster environment on member galaxies have been established. These effects are manifest in the amount and distribution of gas in cluster spirals, the luminosity and light distributions within galaxies and segregation of morphological types and some other effects. Surprisingly, few has been done concerning the dynamics of cluster galaxies. Rubin, Whitmore \& Ford (1988) and Whitmore, Forbes \& Rubin (1988; hereafter collectively referred to as RWF); Distefano et al. (1990); Amram et al. (1992,1993a, 1993b) have observed optical rotation curves (RCs) of cluster galaxies. Their conclusions differ substantially. RWF presented evidence for a correlation in the sense that inner cluster spirals tended to have falling $\mathrm{RCs}$, unlike those of outer cluster spirals or the great majority of field galaxies. This correlation suggested to RWF that inner cluster environment can strip away some fraction of the mass in the outer halo of a spiral galaxy or, alternatively, may not allow the halo to form. Distefano et al. (1990) pointed out that the case of Virgo cluster is quite ambiguous. From a sample of 21 spirals, Amram et al. (1993a) found no significant evidence for anything more than a small influence of the cluster environment. From a more complete sample, especially in the very central parts of clusters, our present conclusion is that spirals in clusters do not have decrasing RCs within the optical radius. 


\section{Observations and data reduction}

The observations have been made between November 1989 and April 1991 using the $3.6 \mathrm{~m}$ CFHT Telescope (Amram et al., 1991; Amram et al., 1993) and between June 1992 and May 1993 at the $3.6 \mathrm{~m}$ ESO Telescope in La Silla (Chili). The instrument PALILA has been used at CFHT and CIGALE (CInématique des GALaxiEs) at ESO. Both instruments are attached at the Cassegrain focus of the telescope and are basically composed of a focal reducer (bringing the original $f / 8$ focal ratio of the Cassegrain focus to $f / 2$ ), and a scanning FabryPerot interferometer. A CCD was used at CFHT while an IPCS (2-D photoncounting) system was used at ESO. The basic principles of this instrument are described in Amram et al., 1991.

Table 1. Observational parameters.

\begin{tabular}{|c|c|c|}
\hline & CFHT(CCD PHX1) & ESO(IPCS) \\
\hline Telescope diameter $(\mathrm{m})$ & 3.60 & 3.60 \\
\hline Line Observed $(\dot{A})$ & $\mathrm{H} \alpha[6563]$ & $\mathrm{H} \alpha[6563]$ \\
\hline Calibration Wavelength $(\AA)$ & $\mathrm{Ne}[6599]$ & $\mathrm{Ne}[6599]$ \\
\hline FWHM ranges of the Interference filters $(\AA)$ & $10-15$ & $10-15$ \\
\hline Interference Free Spectral Range (km. $\left.\star^{-1}\right)$ & 257 & 384 \\
\hline Finesse & 12 & 12 \\
\hline Number of Scanning Steps & 24 & 24 \\
\hline Sampling Step $\left(\mathrm{km} . \mathrm{s}^{-1}\right)$ & 11 & 16 \\
\hline Typical time exposure (hour) & $1 \mathrm{~h} 30$ & $1 \mathrm{~h} 30^{\prime}$ \\
\hline Typical elementary exposure (seconde) & $300 \mathrm{~s}$ & 5s \\
\hline Detector quantum efficiency at $\mathrm{H} \alpha$ & 0.40 & 0.12 \\
\hline Detector read out noise ( $\left.\mathrm{e}^{-} . \mathrm{rms}\right)$ & 7 & 0 \\
\hline Total Field $(n)^{2}$ & $294 \times 294$ & $230 \times 230$ \\
\hline Frame Format $(p x)^{2}$ & $540 \times 540$ & $256 \times 256$ \\
\hline Pixel Size $(")^{2}$ & $1.14 \times 1.14$ & $0.91 \times 0.91$ \\
\hline
\end{tabular}

Table 2. Sample.

\begin{tabular}{llll}
\hline Cluster & \multicolumn{2}{c}{$N^{(a)}$ Cluster } & $N^{(a)}$ \\
\hline Pegasus & 7 & Coma & 5 \\
\hline Cancer & 7 & DC 1842-63 & 4 \\
\hline A 262 & 6 & A 1367 & 2 \\
\hline Hercules & 5 & A 539 & 2
\end{tabular}

${ }^{(a)}$ Number of galaxies observed

The data reduction procedure has been extensively described in Amram et al. (1991). Both CCD and IPCS (2-D photon-counting camera) have been used. Indeed, despite the fact that the quantum efficiency of the IPCS is 2 to 3 times lower than that of a CCD, using an IPCS, one benefits of the multiplex advantages (fast scanning of the Fabry-Perot). Table 1 summarizes the main observational parameters.

Figure 1 illustrates the $\mathrm{H} \alpha$ image and velocity field of a typical galaxy (DC 24 in the Southern cluster of galaxy DC 1842-63, observed at ESO, May 1993). 

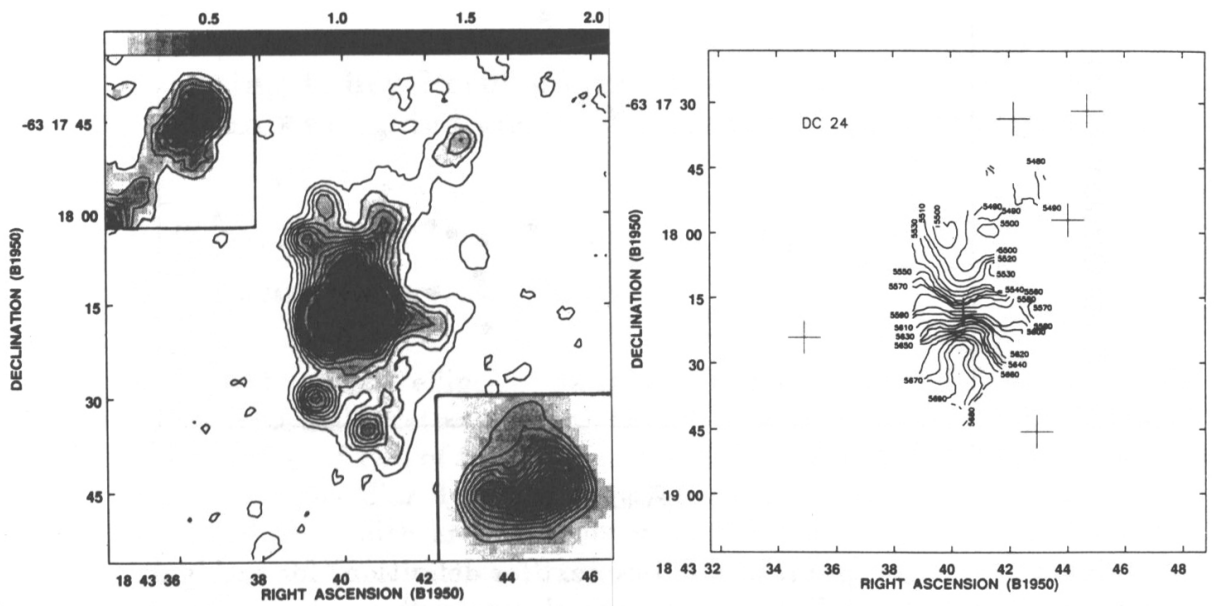

Figure 1. LEFT : $\mathrm{H} \alpha$ image of DC 24. This map is built from the scanning sequence by integrating the flux found in the $\mathrm{H} \alpha$ line for each profile. Contours levels are linearly spaced but with a jump in the step level at $15 \sigma$ level. The left insert shows an 1.5 times enlargement of the galaxy NW region. The right insert shows an enlargement of the galaxy center. RIGHT : Isovelocity contours drawn from $1013 \mathrm{H} \alpha$ profiles superimposed to a photograph.

The rotation curve and mass model of this galaxy as well as a brief discussion is given in Amram et al. (1994).

\section{Results and discussion}

The parameter OG (Outer Gradient) is used to define the slope of the RCs. (OG $\equiv$ outer gradient of the $\mathrm{RC}$, defined as the percentage increase of the RC between $0.4 R_{25}$ and $0.8 R_{25}$, normalized to the maximum rotational velocity $V_{\text {rot }}^{\max }$ ) OG is plotted versus the distance to the center of the different clusters in $\mathrm{Mpc}$ on figure 2. It is clear from this plot that RCs have roughly the same behaviour close to the center as in the outer parts of the clusters.

Spiral galaxies located in the central part of clusters do not have decreasing RCs within the optical radius. Furthermore, the entire sample of galaxies do not present any correlation between the slope of the RCs (OG) and the radial location of the galaxy in the cluster. We are presently looking at the $M / L$ dependancy with the cluster radius.

Nevertheless, the sample presented here could be affected by a selection effect in the sense that most of the galaxies we've observed are late-type spirals while galaxies in central parts of clusters are mainly eraly-type galaxies. (Indeed, in order to be able to compute a RC from $\mathrm{H \alpha}$ emission lines, one are lead to select late-type galaxies). Assuming that environmental effects on the mass distribution of galaxies could have occured only in central cluster regions (typically within $1 \mathrm{Mpc}$ ). One needs to observe as much galaxies as possible in 


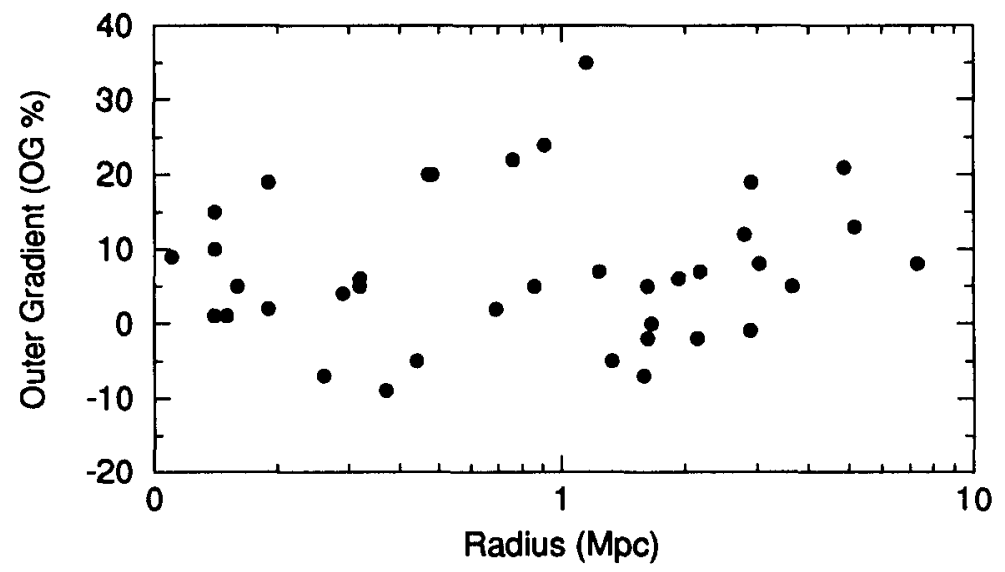

Figure 2. Outer gradient OG (see text for definition) for each galaxy versus its projected distance from the cluster center.

these central regions. Nonetheless there are only a few late observable there in $\mathrm{H} \alpha$ but also they may be located there simply because of projection effects.

\section{References}

Amram, P., Balkowski, C., Marcelin et al. 1994, XXIXth Rencontre de Moriond, Méribel, Savoie, France - 12-19 March 1994 (in press)

Amram, P., Boulesteix, J., Georgelin, Y.P., Georgelin, Y.M., Laval, A., Le Coarer, E., \& Marcelin, M. 1991, The Messenger 64, 44

Amram, P., Le Coarer, E., Marcelin, M., Balkowsky, C., Sullivan III, W.T., \& Cayatte, V. 1992, A\&AS, 94, 175

Amram, P., Marcelin, M., Balkowsky, C., Cayatte, V., Sullivan III, W.T., \& Le Coarer, E. 1994, A\&AS, 103, 5

Amram, P. Sullivan, W.T., Balkowski, C., Marcelin, M., \& Cayatte, V. 1993, ApJ, 403, L59

Distefano, A., Rampazzo, R., Chincarini, G., \& de Souza, R. 1990, A\&AS, 86, 7

Rubin, V.C., Whitmore, B.C. \& Ford, W.K. Jr 1988, ApJ, 333, 522 (RWF)

Whitmore, B.C., Forbes, D.A. \& Rubin, V.C. 1988, ApJ, 333, 542 (RWF) 